\title{
Editorial: Emerging New Tests and Their Impact Upon the Practice of Reproductive Genetics
}

\begin{abstract}
Kwok-yin Leung ${ }^{1,2 * t}$, Antoni Borrell ${ }^{3 \dagger}$, Mark I. Evans ${ }^{4,5+}$ and Ming Chen ${ }^{6,7,8,9,10 * t}$
${ }^{1}$ Department of Obstetrics and Gynecology, University of Hong Kong, Hong Kong, China, ${ }^{2}$ Maternal Fetal Medicine Center, Gleneagles Hospital Hong Kong, Hong Kong, China, ${ }^{3} \mathrm{BCN}$ atal, Hospital Clínic Barcelona, Universitat de Barcelona, Barcelona, Spain, ${ }^{4}$ Comprehensive Genetics, New York, NY, United States, ${ }^{5}$ Department of Obstetrics and Gynecology, Icahn School of Medicine at Mt. Sinai, New York, NY, United States, ${ }^{6}$ Department of Genomic Medicine, Changhua Christian Hospital Medical Center, Changhua, Taiwan, ${ }^{7}$ College of Medicine, National Chung Hsing University, Taichung, Taiwan, ${ }^{8}$ Department of Obstetrics and Gynecology, College of Medicine, and Hospital, National Taiwan University, Taipei, Taiwan, ${ }^{9}$ Department of Medical Science, National Tsing Hua University, Hsinchu, Taiwan, ${ }^{10}$ Department of Biomedical Science, Dayeh University, Changhua, Taiwan
\end{abstract}

Keywords: reproductive genetics, aneuploidy screening, expanded carrier screening, chromosomal microarray analysis, whole-exome sequencing, balanced chromosomal abnormalities, early pregnancy loss, preimplantation genetic testing

Editorial on the Research Topic

Emerging New Tests and Their Impact Upon the Practice of Reproductive Genetics

\section{OPEN ACCESS}

Edited and reviewed by: Thomas Liehr, Friedrich Schiller University Jena, Germany

*Correspondence:

Kwok-yin Leung kyleungog@gmail.com

Ming Chen

mingchenmd@gmail.com

${ }^{\dagger}$ These authors have contributed equally to this work

Specialty section:

This article was submitted to Human and Medical Genomics,

a section of the journal

Frontiers in Genetics

Received: 03 December 2021 Accepted: 03 December 2021 Published: 17 December 2021

Citation:

Leung K-y, Borrell A, Evans MI and Chen $M$ (2021) Editorial: Emerging New Tests and Their Impact Upon the Practice of Reproductive Genetics. Front. Genet. 12:828202. doi: $10.3389 /$ fgene.2021.828202
There has been a geometric explosion in genetic capabilities such that currently a wide variety of prenatal screening and diagnostic testing for fetal chromosomal abnormalities are available. Ideally, all woman should be counseled in each pregnancy about the benefits and limitations of available testing (American College of Obstetricians and Gynecologists' Committee on Practice Bulletins-Obstetrics, 2020). In countries where equity and budgets are prioritized over personal choice by the various National Health Services, testing is offered only when a specific risk cut-off is reached. In the present issue, Antoni Borrell enlightens us on the impact of health education on informed decision. A randomized controlled trial was conducted on 160 pregnant women undergoing first trimester aneuploidy screening (Miño et al.). After receiving an extensive counseling, more women opted for an invasive prenatal testing while less women opted for the first trimester combined test and cell free DNA testing, as compared to those without extensive counseling (Miño et al.).

Recently, American College of Medical Genetics and Genomics (ACMG) recommends a consistent and equitable approach for offering carrier screening to all women during pregnancy or before conception (Gregg et al., 2021). Next-generation sequencing (NGS) allows identification of sequence variants across many genes and hence screening for multiple genetic conditions at the same time. A questionnaire survey involving 623 Chinese pregnant women and 300 nonpregnant women to study views and acceptance of expanded carrier screening (ECS) was reported in the present issue (Cheng et al.). Although more non-pregnant women accepted ECS compared to pregnant women (70.7 vs. $61.2 \%)$, fewer non-pregnant women heard about ECS than pregnant women (32.3 vs. 42. $4 \%)$. The majority of them showed a lack of understanding about ECS despite being given pamphlets (Cheng et al.). It is important to improve women's understanding of reproductive risk before making an informed decision, especially the issue related to false-negatives, of which the readers can refer to a recent excellent review (Evans et al., 2021).

While prenatal chromosomal microarray analysis (CMA) is a current standard genetic testing, prenatal whole exome sequencing and eventually whole genome sequencing are emerging technologies that will become front line tests in the future. CMA can detect major chromosomal imbalances as well as copy number variations that are too small to be detected by traditional 
karyotyping. Diagnostic sequencing is useful for evaluation of fetuses for whom CMA is uninformative. It may be offered concurrently according to accepted practice guidelines. CMA is less optimal than sequencing for the presenting fetal phenotype according to expert genetic opinion (International Society for International Society for Prenatal Diagnosis, 2018). Interpretation of variants found by sequencing is challenging because there is currently limited genotype-phenotype correlation in the prenatal setting. A case report in the present issue illustrates challenges of prenatal counseling due to the complexity of genomic data.

The prenatal identification of a baby with a MECP2 missense mutation and 15q11.2 microduplication in a family with a child having developmental epileptic encephalopathy associated with a de novo KCNQ2 mutation was reported (Huang et al.). The authors used an extended segregation analysis including the parents and their relatives to provide further useful information for genetic counseling.

Another limitation of prenatal sequencing is long turn-around time. In the present issue, prenatal diagnosis of two families with recurrent oligohydramnios by rapid trio-whole-exome sequencing (WES) which revealed mutations in the AGT gene within 1 week was reported (Lin et al.). A compound heterozygous mutation with c.856 + 1G > T and c.857-619_ $1269+243$ delinsTTGCCTTGC changes were found in the first family while homozygous c.857-619_1269 + 243delinsTTGCCTTGC mutations were found in the second family. These mutations are associated with autosomal recessive renal tubular dysgenesis which is a severe disorder with an unfavorable outcome. Notably, a few earlier reports from Taiwan had pointed out that the mutant allele c.857$619 \_1269+243$ delinsTTGCCTTGC of the AGT gene is very likely to be a founder variant in the Chinese population, which may aid in the prompter identification of the molecular pathology in that rapid trio-WEs case (Ma et al., 2019; Tseng et al., 2020). Such founder effect alleles are not uncommon in the Chinese population and had been reported previously at other inherited disorders such as the c.509G > A/p.G170D mutation of the PPIB gene causing the osteogenesis imperfecta IX in the Chinese population, and the way to estimate the mutation dating was also reported (Chang et al., 2020; Zhu et al.).

Recently, whole-genome sequencing (WGS) has been used to delineate the breakpoints of balanced chromosomal abnormalities (BCA) which have no visible gain or loss of genetic material at cytogenetic resolution (Redin et al., 2017). The use of whole-genome sequencing can detect complexity of BCA that is cryptic to karyotyping, and hence improve prediction of clinical outcomes for balanced rearrangements (Redin et al.,

\section{REFERENCES}

American College of Obstetricians and Gynecologists' Committee on Practice Bulletins-Obstetrics (2020). Committee on Genetics; Society for MaternalFetal MedicineScreening for Fetal Chromosomal Abnormalities: ACOG Practice Bulletin, Number 226. Obstet. Gynecol. 136, e48-e69. doi:10.1097/ AOG.0000000000004084
2017). In the present issue, a pilot project was reported, using short read genome sequencing (GS) to retrospectively resequence ten prenatal subjects with de novo BCAs (Yu et al.). GS enabled accurate identification of all breakpoints in these ten cases, revised the conventional karyotype results in nine cases, and provided additional information and changed the interpretation of the BCAs in four cases ( $\mathrm{Yu}$ et al.). These results show us the importance of precisely delineation of breakpoints as BCAs are associated with neurodevelopmental disorders including intellectual disability and autism spectrum disorder.

Chromosome analysis of both couples with recurrent pregnancy loss (RPL) and products of conception (POC) is most informative in the investigation of possible genetic causes of RPL (Papas and Kutteh, 2021). Parental karyotyping can detect translocation carriers in 2-5\% of RPL couples (Papas and Kutteh, 2021), while POC can demonstrate chromosomal anomalies in half of the cases. In the present issue, a new stepwise molecular work-up to diagnose chromosomal abnormalities in women who have had one or more early pregnancy losses was reported (Pauta et al.). This new molecular work-up included two quantitative fluorescence PCR (QF-PCR) rounds, and a high-resolution SNParray in those cases with normal QF-PCR results. QF-PCR and CMA can overcome the pitfalls of conventional karyotyping including culture failure and submicroscopic abnormalities. Besides, the authors also proposed transcervical chorionic villus sampling (CVS) during surgical or before medical uterine evacuation to avoid maternal contamination. They found that transcervical CVS was more effective in the retrieval of embryonic tissue for chromosome analysis than examining POC after evacuation (Pauta et al.).

NGS after trophectoderm (TE) biopsy have been used for preimplantation genetic testing for aneuploidies (PGT-A) because of its high sensitivity (Popovic et al., 2020). Compared to CMA, NGS may detect more chromosome mosaicism in blastocysts which remains a perpetual diagnostic and clinical dilemma (Popovic et al., 2020). In the present issue, a retrospective cohort study on PGT-A from 4,036 blastocysts with NGS was reported (Chuang et al.). The authors explored whether the incidence of mosaicism for individual chromosome in blastocysts is correlated with chromosome length, highlighting the complex mechanisms of causing mosaicism in blastocysts.

\section{AUTHOR CONTRIBUTIONS}

All authors listed have made a substantial, direct, and intellectual contribution to the work and approved it for publication.

Chang, T.-Y., Chung, I.-F., Wu, W.-J., Chang, S.-P., Lin, W.-H., Ginsberg, N. A., et al. (2020). Whole Exome Sequencing with Comprehensive Gene Set Analysis Identified a Biparental-Origin Homozygous c.509G>A Mutation in PPIB Gene Clustered in Two Taiwanese Families Exhibiting Fetal Skeletal Dysplasia during Prenatal Ultrasound. Diagnostics 10, 286. doi:10.3390/diagnostics 10050286

Evans, M. I., Chen, M., and Britt, D. W. (2021). Understanding False Negative in Prenatal Testing. Diagnostics 11, 888. doi:10.3390/diagnostics 11050888 
Gregg, A. R., Aarabi, M., Klugman, S., Leach, N. T., Bashford, M. T., Goldwaser, T., et al. (2021). \& ACMG Professional Practice and Guidelines CommitteeScreening for Autosomal Recessive and X-Linked Conditions during Pregnancy and Preconception: a Practice Resource of the American College of Medical Genetics and Genomics (ACMG). Genet. Med. 23, 1793-1806. doi:10.1038/s41436-021-01203-Z

International Society for Prenatal Diagnosis (2018). Society for Maternal and Fetal Medicine; Perinatal Quality FoundationJoint Position Statement from the International Society for Prenatal Diagnosis (ISPD), the Society for Maternal Fetal Medicine (SMFM), and the Perinatal Quality Foundation (PQF) on the Use of Genome-wide Sequencing for Fetal Diagnosis. Prenat. Diagn. 38, 6-9. doi:10.1002/pd.5195

Ma, G. C., Chen, Y. C., Wu, W. J., Chang, S. P., Chang, T. Y., Lin, W. H., et al. (2019). Prenatal Diagnosis of Autosomal Recessive Renal Tubular Dysgenesis with Anhydramnios Caused by a Mutation in the AGT Gene. Diagnostics 9, 185. doi:10.3390/diagnostics9040185

Papas, R. S., and Kutteh, W. H. (2021). Genetic Testing for Aneuploidy in Patients Who Have Had Multiple Miscarriages: A Review of Current Literature. Tacg 14, 321-329. doi:10.2147/TACG.S320778

Popovic, M., Dhaenens, L., Boel, A., Menten, B., and Heindryckx, B. (2020). Chromosomal Mosaicism in Human Blastocysts: the Ultimate Diagnostic Dilemma. Hum. Reprod. Update 26, 313-334. doi:10.1093/humupd/dmz050

Redin, C., Brand, H., Collins, R. L., Kammin, T., Mitchell, E., Hodge, J. C., et al. (2017). The Genomic Landscape of Balanced Cytogenetic Abnormalities
Associated with Human Congenital Anomalies. Nat. Genet. 49, 36-45. doi:10.1038/ng.3720

Tseng, M.-H., Huang, S.-M., Huang, J.-L., Fan, W.-L., Konrad, M., Shaw, S. W., et al. (2020). Autosomal Recessive Renal Tubular Dysgenesis Caused by a Founder Mutation of Angiotensinogen. Kidney Int. Rep. 5, 2042-2051. doi:10.1016/j.ekir.2020.08.011

Conflict of Interest: The authors declare that the research was conducted in the absence of any commercial or financial relationships that could be construed as a potential conflict of interest.

Publisher's Note: All claims expressed in this article are solely those of the authors and do not necessarily represent those of their affiliated organizations, or those of the publisher, the editors, and the reviewers. Any product that may be evaluated in this article, or claim that may be made by its manufacturer, is not guaranteed or endorsed by the publisher.

Copyright (c) 2021 Leung, Borrell, Evans and Chen. This is an open-access article distributed under the terms of the Creative Commons Attribution License (CC BY).

The use, distribution or reproduction in other forums is permitted, provided the original author(s) and the copyright owner(s) are credited and that the original publication in this journal is cited, in accordance with accepted academic practice. No use, distribution or reproduction is permitted which does not comply with these terms. 